\title{
Insuficiência renal avaliada pela creatinina e a eritropoiese
}

\author{
Renal insufficiency assessed by creatinine and erythropoiesis \\ Insuficiencia renal evaluada por creatinina y eritropoyesis
}

Bruno Lobascz Barbosa

ORCID: https://orcid.org/0000-0002-3713-2301 Centro de Estudos Superior dos Campos Gerais, Brasil E-mail: brunolobascz20@ outlook.com

Geraldo Ricardo da Silva

ORCID: https://orcid.org/0000-0001-8961-7506 Centro de Estudos Superior dos Campos Gerais, Brasil E-mail: ge2ricardo68@gmail.com

Vânia Regina Martins

ORCID: https://orcid.org/0000-0002-8983-7071 Centro de Estudos Superior dos Campos Gerais, Brasil E-mail: varmartins@gmail.com

Rosana Letícia Rosa

ORCID: https://orcid.org/0000-0002-8983-7071 Centro de Estudos Superior dos Campos Gerais, Brasil E-mail: rosanaletícia@ @otmail.com

Robson Schimandeiro Novak

ORCID: https://orcid.org/0000-0002-6997-3260 Centro de Estudos Superior dos Campos Gerais, Brasil E-mail: robsonnovak@hotmail.com

Diego Jose Schebelski

ORCID: https://orcid.org/0000-0001-8647-6760 Centro de Estudos Superior dos Campos Gerais, Brasil E-mail: diego.ski@hotmail.com

\begin{abstract}
Resumo
A Insuficiência Renal Crônica é um problema de saúde pública por se tratar de uma doença silenciosa multifatorial em que o diagnóstico normalmente é tardio e irreversível. O objetivo deste trabalho foi verificar o quanto a insuficiência renal determinada pela dosagem de creatinina acima dos valores de referência pode estar relacionada com a anemia nesses pacientes. Foram selecionadas todas as 4951 requisições de exames registradas no banco de dados de um laboratório de Reserva-PR, no período de janeiro a dezembro de 2020 que, após aplicados os critérios de inclusão e exclusão restaram 105 a serem analisadas. Os critérios de inclusão adotados foram: pacientes que realizaram hemograma e creatinina na mesma requisição, com a faixa etária entre 18 a 60 anos, valores da dosagem de creatinina acima de $1,3 \mathrm{mg} / \mathrm{dL}$. Os critérios de exclusão foram: pacientes com menos de 18 anos ou mais de 60 anos, com creatinina menor de $1,3 \mathrm{mg} / \mathrm{dL}$ e pacientes repetidos com requisições diferentes. Da amostra de 105 pacientes, 23,81\% (25) eram anêmicos, dos quais 32\% (8) mulheres e 68\% (17) homens. Obteve-se que $75 \%$ das anemias nas mulheres e 64,70 \% entre os homens eram do tipo normocíticas normocrômicas. A doença renal crônica acomete principalmente idosos do sexo masculino e a anemia é frequente nesses pacientes. A creatinina é um biomarcador importante e pode ser utilizado como ferramenta coadjuvante em diagnóstico de disfunções renais.
\end{abstract}

Palavras-chave: Insuficiência Renal Crônica; Hemograma; Anemia.

\begin{abstract}
Chronic Kidney Failure is a public health problem because it is a silent multifactorial disease in which diagnosis is usually late and irreversible. The aim of this study was to verify how renal failure determined by creatinine levels above reference values may be related to anemia in these patients. All 4951 exam requests registered in the database of a laboratory in Reserva-PR, from January to December 2020, were selected. After applying the inclusion and exclusion criteria, 105 remained to be analyzed. The inclusion criteria adopted were: patients who underwent blood count and creatinine in the same request, aged between 18 and 60 years, creatinine dosage values above $1.3 \mathrm{mg} / \mathrm{dL}$. Exclusion criteria were: patients under 18 years or over 60 years, with creatinine less than $1.3 \mathrm{mg} / \mathrm{dL}$ and repeat patients with different requirements. Of the sample of 105 patients, $23.81 \%$ (25) were anemic, of which 32\% (8) were women and $68 \%$ (17) were men. It was found that $75 \%$ of anemia in women and $64.70 \%$ in men were of the normocytic normochromic type. Chronic kidney disease mainly affects elderly males and anemia is frequent in these
\end{abstract}


patients. Creatinine is an important biomarker and can be used as an adjunct tool in the diagnosis of kidney dysfunction.

Keywords: Chronic Kidney Failure; Blood count; Anemia.

\begin{abstract}
Resumen
La insuficiencia renal crónica es un problema de salud pública porque es una enfermedad multifactorial silenciosa en la que el diagnóstico suele ser tardío e irreversible. El objetivo de este estudio fue verificar cómo la insuficiencia renal determinada por niveles de creatinina por encima de los valores de referencia puede estar relacionada con la anemia en estos pacientes. Se seleccionaron las 4951 solicitudes de examen registradas en la base de datos de un laboratorio en Reserva-PR, de enero a diciembre de 2020. Luego de aplicar los criterios de inclusión y exclusión, quedaron 105 por analizar. Los criterios de inclusión adoptados fueron: pacientes a los que se les realizó hemograma y creatinina en la misma solicitud, con edades comprendidas entre 18 y 60 años, valores de dosis de creatinina superiores a 1,3 mg / dL. Los criterios de exclusión fueron: pacientes menores de 18 años o mayores de 60 años, con creatinina inferior a $1,3 \mathrm{mg} / \mathrm{dl}$ y pacientes reincidentes con diferentes necesidades. De la muestra de 105 pacientes, el 23,81\% (25) eran anémicos, de los cuales el 32\% (8) eran mujeres y el 68\% (17) eran hombres. Se encontró que el 75\% de las anemias en mujeres y el 64,70\% en hombres eran de tipo normocítico normocrómico. La enfermedad renal crónica afecta principalmente a varones ancianos y la anemia es frecuente en estos pacientes. La creatinina es un biomarcador importante y se puede utilizar como herramienta complementaria en el diagnóstico de disfunción renal.
\end{abstract}

Palabras clave: Insuficiencia Renal Erónica; Conteo de glóbulos; Anemia.

\title{
1. Introduçãa
}

Com o aumento da expectativa de vida das pessoas, as doenças crônicas se tornaram motivo de preocupação para os serviços de saúde pública nas últimas décadas. A obesidade, a hipertensão arterial e o diabetes mellitus se destacam como os principais gatilhos da doença renal crônica, a qual possui origem silenciosa, proporcionando, na maioria dos casos um diagnóstico tardio, limitando assim o sucesso do tratamento (Crews, Bello \& Saadi, 2019).

A fundação Kidney Disease Quality Outcome Iniciative (K/DOQI) sugere a definição de doença renal crônica (DRC) como lesão renal ou Taxa de Filtração Glomerular (TFG) $<60 \mathrm{~mL} / \mathrm{min} / 1,73 \mathrm{~m}^{2}$ durante um período de 3 meses ou mais, mesmo sem causa definida. A estimativa da TFG pode ser obtida pela creatina sérica adequada através da equação do estudo de modificação da dieta em doenças renais (MDRD) ou a fórmula de Cockcroft-Gaut (CG) (Bukabau et al., 2019). A avaliação da filtração glomerular é essencial para determinar o funcionamento dos rins tanto em indivíduos normais, quanto para pacientes com doença renal. Esse processo pode ser definido pela dosagem de creatinina sérica (Bastos et al., 2004).

Os índices de creatinina considerados de referência são de $0,7 \mathrm{mg} / \mathrm{dL}$ a 1,3 mg/dL. Quando a função renal entra em declínio, a capacidade de eliminar resíduos tóxicos fica deficiente, ocorrendo a elevação dos níveis séricos de creatinina. Em alguns casos, o agravo das funções renais compromete a homeostasia de síntese de eritropoietina nos rins e, assim, interfere na produção de eritrócitos, resultando em anemia crônica (Ribeiro-Alves \& Gordan, 2014).

Os valores elevados de creatinina podem ser utilizados como biomarcadores das funções renais, contribuindo para o diagnóstico de insuficiência renal. A identificação e o tratamento precoce da anemia em pacientes com doença renal crônica podem melhorar a qualidade de vida desses pacientes e evitar consequências como os problemas cardiovasculares, entre outros (Bueno \& Frizzo, 2014; Ribeiro, 2017).

Sobre o impacto da doença renal na população, a Sociedade Brasileira de Nefrologia (SBN) alerta que, quando os rins apresentam algum tipo de ineficiência, a doença pode evoluir de forma que haja necessidade de tratamento dialítico. No Brasil, a cada ano, aproximadamente 21 mil pacientes precisam iniciar hemodiálise ou diálise peritoneal (Abreu, Riella \& Nascimento, 2020). O estudo realizado por Sesso et al. (2017) apontou um aumento no número de centros de diálise ativos para atender a demanda, os quais tiveram uma taxa de ocupação de $84 \%$, sendo constatado também que, em julho de 2016 , havia um número estimado de 122.825 pacientes em tratamento por diálise.

A fisiopatologia da doença renal crônica possui como característica importante a redução no número de células peritubulares renais e, por consequência, a diminuição da síntese de eritropoietina (EPO), a qual tem a função de estimular a 
eritropoiese na medula óssea para normalizar a população de eritrócitos no caso de hipóxia e, portanto suprir as necessidades de oxigênio nos tecidos (Souza \& Gomes, 2017; Hoffbrand \& Moss, 2018). Na medida em que a função renal decai, ocorre o surgimento de anemia em pacientes renais crônicos, comprometendo a vitalidade do organismo, bem como interferindo na qualidade de vida do indivíduo acometido, sendo possível a evolução para problemas cardiovasculares e morte prematura (Silva \& Couto, 2016).

A recomendação para o diagnóstico de anemia em pacientes adultos portadores de doença renal crônica em qualquer estágio da doença é de dosagem de hemoglobina que, para homens não deve ser menor que $13 \mathrm{~g} / \mathrm{dL}$ e para mulheres, menor do que $12 \mathrm{~g} / \mathrm{dL}$. Na insuficiência renal crônica, devido a fisiopatologia da doença, as anemias se manifestam como normocíticas normocrômicas, principalmente em função da diminuição da síntese de eritropoietina que ocorre quando a taxa de filtração glomerular atinge índices menores que $70 \mathrm{~mL} / \mathrm{min} / 1,73 \mathrm{~m}^{2}$ para homens e $50 \mathrm{~mL} / \mathrm{min} / 1,73 \mathrm{~m}^{2}$ para mulheres. Quando o achado morfológico da anemia é diferente, outras causas devem ser investigadas (Ribeiro-Alves \& Gordan, 2014).

A síntese inadequada de eritropoietina é um fator determinante no desenvolvimento de anemia nesses pacientes, sendo que o tratamento com eritropoietina recombinante exógena nesse tipo de doença melhora os índices hematimétricos e a qualidade de vida dos pacientes (Ribeiro, 2017).

Em consequência de tratamento com intervenções laboratoriais, perdas gastrintestinais imperceptíveis e intervenções cirúrgicas, pode ocorrer em algum momento deficiência de ferro. Tal avaliação deve ser constatada com dosagem de ferritina e outros exames para a avaliação laboratorial do ferro do paciente. Os processos inflamatórios são comuns na doença renal crônica e, nessa condição, pode apresentar um alto estoque de ferritina sem que haja estoque adequado de ferro. Para avaliar a resposta da medula óssea ao tratamento com medicamentos estimuladores de eritropoiese é adequado utilizar na prática clínica, contadores automatizados que forneçam o parâmetro de conteúdo de hemoglobina em reticulócitos (CHr) (Ribeiro, 2017).

Esse trabalho teve como finalidade verificar o quanto a insuficiência renal determinada pela dosagem de creatinina acima dos valores de referência pode estar relacionada com a anemia nesses pacientes.

\section{Metodologia}

Estudo observacional, analítico, de corte transversal, de natureza básica (Bruce, 2019). A coleta de dados foi realizada no período de janeiro a dezembro de 2020, a partir do banco de dados do laboratório Bio-Análises localizado em Reserva - PR, que inicialmente contava com 4951 pacientes. Após aplicados os critérios de inclusão e exclusão restaram 105 pacientes para análise. A amostra foi composta por pacientes femininos e masculinos com faixa etária entre 18 e 60 anos.

Os critérios de inclusão adotados foram: pacientes que realizaram hemograma e creatinina na mesma requisição, com a faixa etária entre 18 e 60 anos, creatinina acima de $1,3 \mathrm{mg} / \mathrm{dL}$. Os critérios de exclusão foram: pacientes com menos de 18 anos ou mais de 60 anos, com creatinina menor de $1,3 \mathrm{mg} / \mathrm{dL}$ e pacientes com a creatinina acima de $1,3 \mathrm{mg} / \mathrm{dL}$ repetidos com requisições diferentes, permanecendo apenas o resultado onde a creatinina estava mais elevada.

O hemograma foi realizado no equipamento ABX Pentra 60 e as variáveis coletadas foram eritrócitos, hemoglobina, hematócrito, Volume Corpuscular Médio (VCM), Hemoglobina Corpuscular Média (HCM), Concentração de Hemoglobina Corpuscular média (CHCM) e Red Cell Distribution Width (RDW). A dosagem de creatinina sérica foi realizada pela metodologia Enzimática Colorimétrica no equipamento Envoy 500+. Todos os equipamentos tinham controle de qualidade interno e externo de acordo com a RDC ANVISA 302/2005.

Os valores de referência utilizados para comparar os dados da hematologia foram obtidos do livro Hemograma: como fazer e interpretar (Oliveira, 2011), e para os valores de referência da bioquímica foi utilizado o livro Princípios de Bioquímica (Lehninger et al., 2017). 
Foi utilizado o software EPI Info Versão 7.1.5.2 para tabulação dos resultados, onde os dados foram comparados e analisados. Utilizou-se também o software Microsoft Office Excel 2016 para construção dos gráficos e tabelas. Os dados foram apresentados como números absolutos e frequências.

Este estudo respeitou os preceitos éticos e foi enviado ao Comitê de Ética e Pesquisa, recebendo aprovação pelo parecer número 4.587.712.

\section{Resultados e Discussão}

Após a análise dos dados obtidos no banco de dados do laboratório Bio-Análises verificou-se que a idade média da amostra foi de 48,2 anos ( $\pm 11,02$ anos). A idade média, quando considerado o gênero, foi de 48,12 anos ( $\pm 10,53$ anos) para as mulheres e de 42,10 anos ( $\pm 11,17$ anos) para os homens. A distribuição da idade por faixa etária é apresentada na Tabela 1.

Tabela 1 - Frequência da faixa etária dos indivíduos com creatinina elevada no laboratório Bio-Análises, no período de janeiro a dezembro de 2020 .

\begin{tabular}{l|l|l}
\hline $\begin{array}{l}\text { Faixa Etária } \\
\text { (anos) }\end{array}$ & $\mathbf{N}^{\mathbf{o}}$ & $\boldsymbol{\%}$ \\
\hline $\mathbf{1 8 - 2 9}$ & 8 & 7,62 \\
\hline $\mathbf{3 0 - 3 9}$ & 13 & 12,38 \\
\hline $\mathbf{4 0 - 4 9}$ & 25 & 23,81 \\
\hline $\mathbf{5 0 - 6 0}$ & 59 & 56,81 \\
\hline Total & $\mathbf{1 0 5}$ & $\mathbf{1 0 0 , 0 0}$ \\
\hline
\end{tabular}

Fonte: Autores (2021).

Barreto, Santos \& Vivas (2016), cujo trabalho tratou do perfil hematológico em pacientes renais crônicos, citam que ocorre um maior acometimento da doença renal crônica a partir dos 40 anos. De Miranda et al. (2018), perceberam a prevalência de anemia nos pacientes com doença renal crônica em tratamento de hemodiálise, de modo que relataram que a média de idade da amostra foi de 50,37 $\pm 14,47$ anos, resultados esses, similares aos que foram verificados neste trabalho.

Das 105 amostras estudadas, observou-se que 15,24\% (16) da amostra foi do gênero feminino e 84,76\% (89) do gênero masculino e essa distribuição é apresentada na Figura 1.

Figura 1 - Frequência do gênero dos indivíduos com creatinina elevada no laboratório Bio-Análises, no período de janeiro a dezembro de 2020.

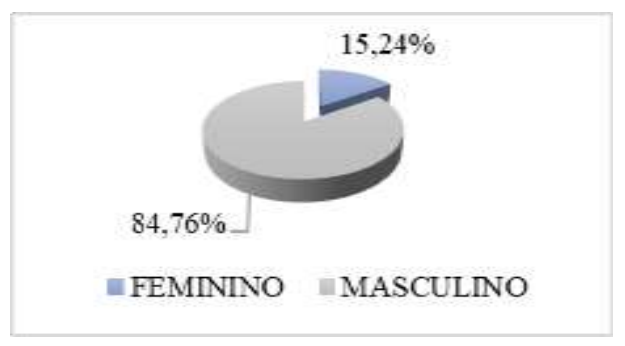

Fonte: Autores (2021).

No trabalho de Sarmento et al. (2018) foram coletados dados de 830 pacientes dos quais, após aplicados os critérios de exclusão, obtiveram 818 prontuários de pacientes renais crônicos da cidade de Fortaleza no Ceará, onde verificou-se que 
61,1\% dos pacientes eram do sexo masculino. Negretti, Mesquita \& Baracho (2014), verificaram o perfil epidemiológico de pacientes renais crônicos em tratamento conservador em um hospital escola do sul de Minas Gerais, onde apenas 50,3\% da população estudada era do sexo masculino. Em Barreto, Santos \& Vivas (2016), 62,5\% da amostra eram homens e De Oliveira Junior \& Caixeta (2018), relatam que os homens são menos frequentes em buscar os serviços de saúde e essa prática contribui para maior incidência da doença em virtude de diagnóstico tardios.

Quando analisado o eritrograma da amostra estudada observou-se que o número de eritrócitos se mostrou fora dos valores de referência em 38,2\% (34) das amostras masculinas e 37,5\% (6) das femininas. Quanto a hemoglobina, 19,10\% (17) dos pacientes masculinos e 50\% (8) dos femininos eram anêmicos. Já para o hematócrito, observou-se 56,25\% (9) mulheres e $31,46 \%$ (28) dos homens com resultado alterado. O VCM nos mostra 10,48\% (11) dos pacientes com microcitose e 1,90\% (2) com macrocitose; o HCM apresentou 10,48\% (11) das amostras hipocrômicas e 9,52\% (10) com HCM acima de 32 pg. Em 19,05\% (20) da amostra verificou-se anisocitose. A distribuição dessas variáveis é apresentada na Tabela 2.

Tabela 2 - Frequências das variáveis do hemograma dos indivíduos com creatinina elevada no laboratório Bio-Análises, no período de janeiro a dezembro de 2020.

\begin{tabular}{l|l|l}
\hline Eritrócitos Masculinos & $\mathbf{N}^{\mathbf{o}}$ & $\mathbf{\%}$ \\
\hline$<4,5 \mathrm{M} / \mu \mathrm{L}$ & 24 & 26,96 \\
\hline $4,5 \mathrm{M} / \mu \mathrm{L}$ a $5,5 \mathrm{M} / \mu \mathrm{L}$ & 55 & 61,80 \\
\hline$>5,5 \mathrm{M} / \mu \mathrm{L}$ & 10 & 11,24 \\
\hline Total & 89 & 100 \\
\hline Eritrócitos Femininos & $\mathbf{N}^{\mathbf{o}}$ & $\%$ \\
\hline$<3,8 \mathrm{M} / \mu \mathrm{L}$ & 3 & 18,75 \\
\hline $3,8 \mathrm{M} / \mu \mathrm{L}$ a $4,8 \mathrm{M} / \mu \mathrm{L}$ & 10 & 62,50 \\
\hline$>4,8 \mathrm{M} / \mu \mathrm{L}$ & 3 & 18,75 \\
\hline Total & 16 & 100,00 \\
\hline Hemoglobina Masculino & $\mathbf{N}^{\mathbf{o}}$ & $\mathbf{\%}$ \\
\hline$<13 \mathrm{~g} / \mathrm{dL}$ & 17 & 19,10 \\
\hline $13 \mathrm{~g} / \mathrm{dL}$ a $17 \mathrm{~g} / \mathrm{dL}$ & 68 & 76,40 \\
\hline$>17 \mathrm{~g} / \mathrm{dL}$ & 4 & 4,50 \\
\hline Total & 89 & 100,00 \\
\hline Hemoglobina Feminino & $\mathbf{N}^{\mathbf{o}}$ & $\mathbf{\%}$ \\
\hline$<12 \mathrm{~g} / \mathrm{dL}$ & 8 & 50,00 \\
\hline $12 \mathrm{~g} / \mathrm{dL}$ a $15 \mathrm{~g} / \mathrm{dL}$ & 7 & 43,75 \\
\hline$>15 \mathrm{~g} / \mathrm{dL}$ & 1 & 6,25 \\
\hline Total & 16 & 100,00 \\
\hline Hematócrito Feminino & $\mathbf{N}^{\mathbf{o}}$ & $\%$ \\
\hline$<36 \%$ & 9 & 56,25 \\
\hline $36 \%$ a 46\% & 7 & 43,75 \\
\hline Total & 16 & 100,00 \\
\hline & & \\
\hline & & \\
\hline & & \\
\hline
\end{tabular}




\begin{tabular}{|c|c|c|}
\hline Hematócrito Masculino & $\mathbf{N}^{\mathbf{o}}$ & $\%$ \\
\hline$<40 \%$ & 24 & 26,97 \\
\hline $40 \%$ a $50 \%$ & 61 & 68,54 \\
\hline$>50 \%$ & 4 & 4,49 \\
\hline Total & 89 & 100,00 \\
\hline VCM & $\mathbf{N}^{\mathbf{o}}$ & $\%$ \\
\hline$<82 \mathrm{fL}$ & 11 & 10,48 \\
\hline $82 \mathrm{fL}$ a $98 \mathrm{fL}$ & 92 & 87,62 \\
\hline$<98 \mathrm{fL}$ & 2 & 1,90 \\
\hline Total & 89 & 100,00 \\
\hline HCM & $\mathbf{N}^{\mathbf{o}}$ & $\%$ \\
\hline$<27 \mathrm{pg}$ & 11 & 10,48 \\
\hline $27 \mathrm{pg}$ a $32 \mathrm{pg}$ & 84 & 80,00 \\
\hline$>32 \mathrm{pg}$ & 10 & 9,52 \\
\hline Total & 105 & 100,00 \\
\hline CHCM & $\mathbf{N}^{\mathbf{o}}$ & $\%$ \\
\hline $30 \%$ a $36 \%$ & 105 & 100,00 \\
\hline Total & 105 & 100,00 \\
\hline RDW & $\mathbf{N}^{\mathbf{o}}$ & $\%$ \\
\hline$<11,5 \%$ & 8 & 7,62 \\
\hline $11,5 \%$ a $14,5 \%$ & 85 & 80,95 \\
\hline$>14,5 \%$ & 12 & 11,43 \\
\hline Total & 105 & 100,00 \\
\hline
\end{tabular}

Fonte: Autores (2021).

Para indivíduos saudáveis do sexo masculino, é considerado como referência uma contagem de hemácias entre 4,5 milhões $/ \mathrm{mm}^{3}$ e 6,1 milhões $/ \mathrm{mm}^{3}$ e para mulheres entre 4,1 e 5,3 milhões $/ \mathrm{mm}^{3}$ (Soares, 2015). Barreto, Santos \& Vivas (2016), observaram em seu trabalho sobre doentes renais crônicos em tratamento dialítico, que o número de hemácias entre os homens variou de 2,04 milhões $/ \mathrm{mm}^{3}$ a 4,86 milhões $/ \mathrm{mm}^{3}$ e para mulheres a variação foi de 1,73 milhões $/ \mathrm{mm}^{3}$ a 4,26 milhões $/ \mathrm{mm}^{3}$, isto é, quase toda amostra estudada por eles mostrou número de eritrócitos abaixo dos valores recomendados. A população estudada neste trabalho apresentou 61,80\% dos homens e 62,50\% das mulheres dentro dos valores de referência. Essa diferença ocorre devido as amostras nos dois trabalhos terem origens diferentes: na primeira, todos os pacientes estavam em 
tratamento dialítico e na amostra desse trabalho, a amostra constituiu-se de pacientes que tinham a creatinina elevada e não faziam hemodiálise no momento da coleta dos dados, portanto pacientes em situação renal menos grave.

Um estudo realizado por Draczevski \& Teixeira, (2011) apontou que a maioria dos doentes renais crônicos estudados que se submeteram a hemodiálise apresentou algum grau de anemia. A presença de anemia é definida quando a dosagem de hemoglobina é menor que $13 \mathrm{~g} / \mathrm{dL}$ para homens e $12 \mathrm{~g} / \mathrm{dL}$ para mulheres (Ribeiro-Alves \& Gordan, 2014). No presente trabalho, a hemoglobina estava dentro dos valores de referência em 76,4 \% da amostra do gênero masculino e $43,75 \%$ no gênero feminino e 25 pacientes, que representam 23,81\% da amostra, eram anêmicos; destes, 32\% (8) eram mulheres e 68\% (17) homens, conforme mostrado na Figura 2.

Figura 2 - Frequência de anemia nos indivíduos com creatinina elevada no laboratório Bio-Análises, no período de janeiro a dezembro de 2020.

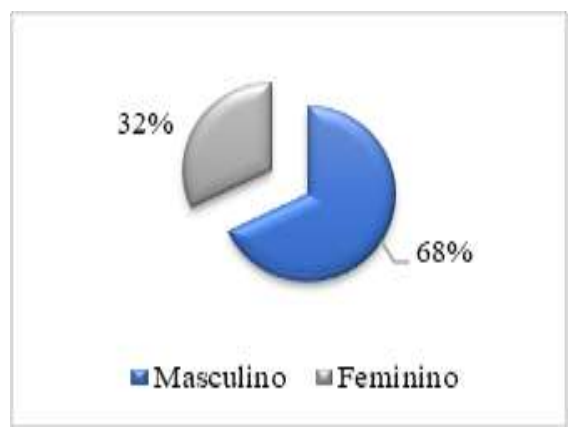

Fonte: Autores (2021).

Em Barreto, Santos \& Vivas (2016) que estudaram 24 indivíduos hemodializados, os homens mostraram uma variação de hemoglobina de 5,8 g/dL a 13,5 g/dL e as mulheres, de 5,2 g/dL a 15,6 g/dL, contudo apenas 2 homens e três mulheres apresentaram hemoglobina dentro dos valores de referência, isso porque provavelmente todos os pacientes estavam em hemodiálise.

Em um trabalho realizado por Bueno \& Frizzo (2014) que avaliaram os parâmetros hematológicos de 45 pacientes em um hospital da região noroeste do Rio Grande do Sul que realizavam tratamento no setor de hemodiálise, constataram um hematócrito < $39 \%$ para homens e < $35 \%$ para mulheres em mais de $90 \%$ da amostra analisada sem grandes alterações durante todo o período do estudo. Já Barreto, Santos \& Vivas (2016) obtiveram uma variação de resultados entre 17,6\% e 41,1\% para homens e 17,6\% a 39,4\% para as mulheres. Em indivíduos saudáveis o valor de referência do hematócrito em homens adultos varia de 40 a $50 \%$ e para as mulheres de 36 a 46\%. Neste trabalho, o gênero masculino demonstrou valores de hematócrito dentro da referência em 68,54\% da amostra já, entre as mulheres 56,25\% delas tinha hematócrito <36\%.

Draczevski \& Teixeira (2011) avaliaram o perfil bioquímico e parâmetros hematológicos em 20 pacientes submetidos à hemodiálise em uma clínica do oeste de Santa Catarina e observaram microcitose na maioria dos pacientes, entretanto, 3 pacientes apresentaram macrocitose; observaram também todos os valores de CHCM dentro da normalidade significando normocromia, característica que predomina na doença renal crônica. Barreto, Santos \& Vivas (2016) apontaram em seu estudo que $96 \%$ dos pacientes apresentaram VCM dentro da normalidade caracterizando a anemia como normocítica.

Nesse trabalho observou-se que 23,8\% (25) dos pacientes eram anêmicos, sendo destes 35,29\% (6) homens com anemia microcítica hipocrômica e 64,70\% (11) com anemia normocítica normocrômica, já entre as mulheres 25\% (2) tinham anemia microcítica hipocrômica e 75\% (6) anemia normocítica normocrômica, conforme mostrado na Tabela 3. 
Tabela 3 - Distribuição de anemias de acordo com o gênero na população estudada no laboratório Bio-Análises, no período de janeiro a dezembro de 2020.

\begin{tabular}{l|c|c}
\hline & Masculino & Feminino \\
\hline Anemia Micrôcítica Hipocromica & $35,29 \%$ & $25 \%$ \\
\hline Anemia Normocítica Normicrômica & $64,70 \%$ & $75 \%$ \\
\hline Total & $\mathbf{1 0 0 , 0 0 \%}$ & $\mathbf{1 0 0 , 0 0 \%}$ \\
\hline
\end{tabular}

Fonte: Autores (2021).

A anemia normocítica normocrômica é um achado frequente na doença renal (Bueno \& Frizzo, 2014; Ribeiro-Alves \& Gordan, 2014; Ribeiro, 2017), e este trabalho também demonstrou frequência semelhante. Soares (2015) verificou que 88\% dos pacientes possuíam anemia normocítica e para Draczevski \& Teixeira (2011), foram 70\% de anêmicos normocíticos.

Após a análise da distribuição dos valores da creatinina elevada por faixa etária e gênero, verificou-se que no sexo feminino a faixa etária de 40 a 49 anos representou 43,75\% (7) com a creatinina acima dos valores de referência, bem como na faixa etária de 50 a 60 anos com a mesma representatividade de 43,75\% (7). No gênero masculino entre 40 e 49 anos, $20,22 \%$ (18) mostraram valores de creatinina fora dos parâmetros considerados nesse trabalho e de 50 a 60 anos, 58,43\% (89). Esses valores mostram que ambos os sexos apresentaram algum grau de comprometimento renal, principalmente após os 40 anos de idade (Tabela 4). O artigo publicado pelo Núcleo Interdisciplinar de Estudos e Pesquisas em Nefrologia (2009), cita que os rins passam a ter alterações anatômicas e alterações de função fisiológica a partir dos 40 anos de idade, principalmente em indivíduos com pesados encargos financeiros e sociais.

Tabela 4 - Distribuição dos resultados de creatinina elevada por faixa etária e gênero no laboratório Bio-Análises no período de janeiro a dezembro de 2020.

\begin{tabular}{c|c|c|c|c|c|c}
\hline \multicolumn{6}{c}{ Creatinina elevada por faixa etária e gênero } \\
\hline \multirow{2}{*}{ Faixa etária } & \multicolumn{2}{|c|}{ Feminino } & \multicolumn{2}{|c}{ Masculino } & \multicolumn{2}{|c}{ Total } \\
\cline { 2 - 7 } & $\mathbf{N}^{\mathbf{0}}$ & $\%$ & $\mathbf{N}^{\mathbf{0}}$ & $\%$ & $\mathbf{N}^{\mathbf{0}}$ & $\mathbf{\%}$ \\
\hline $\mathbf{1 8 - 2 9}$ & 1 & 6,25 & 7 & 7,87 & 8 & $\mathbf{7 , 6 2}$ \\
\hline $\mathbf{3 0 - 3 9}$ & 1 & 6,25 & 12 & 13,48 & 13 & $\mathbf{1 2 , 3 8}$ \\
\hline $\mathbf{4 0 - 4 9}$ & 7 & 43,75 & 18 & 20,22 & 25 & $\mathbf{2 3 , 8 1}$ \\
\hline $\mathbf{5 0 - 6 0}$ & 7 & 43,75 & 52 & 58,43 & 59 & $\mathbf{5 6 , 1 9}$ \\
\hline Total & $\mathbf{1 6}$ & $\mathbf{1 0 0}$ & $\mathbf{8 9}$ & $\mathbf{1 0 0}$ & $\mathbf{1 0 5}$ & $\mathbf{1 0 0}$ \\
\hline & \multicolumn{3}{|c|}{ Fonte: Autores } & $(2021)$ & & \\
\hline
\end{tabular}

Quando avaliado o resultado da dosagem de creatinina elevada em relação a dosagem de hemoglobina de acordo com o gênero, verificou-se que 50\% (8) das mulheres tinham algum grau de comprometimento renal e eram anêmicas e o mesmo ocorreu com 19,10\% (17) dos homens observados. Essa distribuição é apresentada na Tabela 5. 
Tabela 5 - Distribuição dos resultados de creatinina elevada por resultados de dosagem de hemoglobina e gênero no laboratório Bio-Análises, no período de janeiro a dezembro de 2020.

\begin{tabular}{c|c|c}
\hline \multicolumn{3}{c}{ Creatinina elevada feminino } \\
\hline $\begin{array}{c}\text { Hemoglobina } \\
\text { feminino }\end{array}$ & $\mathrm{N}^{\mathbf{0}}$ & $\%$ \\
$<12 \mathrm{~g} / \mathrm{dL}$ & 8 & 50 \\
\hline $12 \mathrm{~g} / \mathrm{dL}$ a $15 \mathrm{~g} / \mathrm{dL}$ & 7 & 43,75 \\
\hline$>15 \mathrm{~g} / \mathrm{dL}$ & 1 & 6,25 \\
\hline Total & $\mathbf{1 6}$ & $\mathbf{1 0 0}$ \\
\hline Creatinina elevada masculino \\
\hline \begin{tabular}{c|c|c} 
Hemoglobina \\
masculino
\end{tabular} & $\mathrm{N}^{\mathbf{0}}$ & $\%$ \\
\hline$<13 \mathrm{~g} / \mathrm{dL}$ & 17 & 19,1 \\
\hline $13 \mathrm{~g} / \mathrm{dL}$ a $17 \mathrm{~g} / \mathrm{dL}$ & 68 & 76,4 \\
\hline$>17 \mathrm{~g} / \mathrm{dL}$ & 4 & 4,49 \\
\hline Total & $\mathbf{8 9}$ & $\mathbf{1 0 0}$ \\
\hline
\end{tabular}

Fonte: Autores (2021).

Bueno \& Frizzo (2014) analisaram o prontuário de 45 pacientes, sendo 31 homens e 14 mulheres que eram submetidos a hemodiálise e realizavam exames de rotina trimestralmente. A média da faixa etária dessa amostra foi de 61 anos e 44 pacientes $(97,8 \%)$ apresentaram anemia durante todo o período do estudo. A creatinina foi outro parâmetro analisado e permaneceu acima dos valores de referência com singela melhora após hemodiálise. Canziani et al. (2006), citam que pacientes portadores de doença renal crônica, que não haviam iniciado ainda a fase de hemodiálise e que apresentaram taxa de depuração entre $10 \mathrm{~mL} / \mathrm{min}$ e $90 \mathrm{~mL} / \mathrm{min}$., já apresentaram algum grau de anemia. Gordino (2019) estudou a epidemiologia da insuficiência renal crônica e anemia associada em adultos na população em geral em Portugal e obteve que a maioria dessa população $(61,54 \%)$ mostrou dosagens de hemoglobina superiores a 12 g/dL e níveis de creatinina entre $0,5 \mathrm{mg} / \mathrm{dL}$ e $1,2 \mathrm{mg} / \mathrm{dL}$. Verificou ainda que, apenas $0,18 \%$ da população apresentou níveis de hemoglobina inferiores a $12 \mathrm{~g} / \mathrm{dL}$ e valores de creatinina acima dos normais. Peixoto et al. (2019), compararam algumas variáveis laboratoriais relacionadas à doença renal crônica e associaram os dados obtidos com a idade e o sexo dos indivíduos estudados. Esse estudo mostrou correlação positiva muito forte entre os pacientes com hemoglobina abaixo do normal e a idade principalmente no grupo de mulheres $(p<0,0001)$. No sexo masculino também foi observada correlação positiva com a idade $(p=0,004)$.

Os dados de distribuição da dosagem de hemoglonina e VCM no laboratório Bio-Análises, de janeiro a dezembro de 2020 estão na Tabela 6 e denota normocitose ou microcitose na grande maioria dos casos, independentemente do gênero. De Oliveira Junior \& Caixeta (2018), citam em seu estudo que em 309 pacientes submetidos à hemodiálise atendidos no laboratório do Hospital São Lucas da cidade de Patos de Minas, que observaram um VCM de 90,58 fL e HCM de 29,60 pg na média total da população, isto é, eram normocíticos normocrômicos. Já De Oliveira Junior et al. (2019), em sua revisão, concluíram que a anemia na doença renal é majoritariamente normocítica normocrômica, contudo em casos mais graves pode se manifestar como microcítica e hipocrômica pela deficiência de ferro. Abenshur (2010) cita que anemia na doença renal 
normalmente é hipoproliferativa e a contagem de células vermelhas na medula óssea é normal ou diminuída confirmando anemia normocítica.

Tabela 6 - Distribuição da dosagem de hemoglonina e VCM no laboratório Bio-Análises no período de janeiro a dezembro de 2020 .

\begin{tabular}{|c|c|c|c|c|c|c|c|c|}
\hline \multirow{3}{*}{$\begin{array}{ll}\begin{array}{l}\text { Dosagem } \\
\text { Hemoglobina }(\mathrm{Hb})\end{array} & \text { de } \\
\text { Hb feminino } & \end{array}$} & \multicolumn{8}{|c|}{ VCM } \\
\hline & \multicolumn{2}{|c|}{$<82 \mathrm{fL}$} & \multicolumn{2}{|c|}{$82 \mathrm{fL}$ a $98 \mathrm{fL}$} & \multicolumn{2}{|c|}{$<98 \mathrm{fL}$} & \multicolumn{2}{|c|}{ Total } \\
\hline & $\mathbf{N}^{\mathbf{o}}$ & $\%$ & $\mathbf{N}^{\mathbf{o}}$ & $\%$ & $\mathbf{N}^{\mathbf{o}}$ & $\%$ & $\mathbf{N}^{\mathbf{o}}$ & $\%$ \\
\hline$<12 \mathrm{mg} / \mathrm{dL}$ & 2 & 25,00 & 6 & 75,00 & 0 & 0,00 & 8 & 100 \\
\hline $12 \mathrm{mg} / \mathrm{dL}$ a $15 \mathrm{mg} / \mathrm{dL}$ & 0 & 0,00 & 7 & 100,00 & 0 & 0,00 & 7 & 100 \\
\hline$>15 \mathrm{mg} / \mathrm{dL}$ & 0 & 0,00 & 1 & 100,00 & 0 & 0,00 & 1 & 100 \\
\hline Total & 2 & 15,38 & 14 & 84,62 & 0 & 0,00 & 16 & 100 \\
\hline Hb masculino & $\mathrm{N}^{\mathrm{o}}$ & $\%$ & $\mathrm{~N}^{\mathrm{o}}$ & $\%$ & $\mathrm{~N}^{\mathrm{o}}$ & $\%$ & $\mathrm{~N}^{\mathrm{o}}$ & $\%$ \\
\hline$<13 \mathrm{mg} / \mathrm{dL}$ & 6 & 35,29 & 11 & 64,71 & 0 & 0,00 & 17 & 100 \\
\hline $13 \mathrm{mg} / \mathrm{dL}$ - $17 \mathrm{mg} / \mathrm{dL}$ & 3 & 4,41 & 64 & 94,12 & 1 & 1,47 & 68 & 100 \\
\hline$>17 \mathrm{mg} / \mathrm{dL}$ & 0 & 0,00 & 3 & 75,00 & 1 & 25,00 & 4 & 100 \\
\hline Total & 9 & 10,11 & 78 & 87,64 & 2 & 2,25 & 89 & 100 \\
\hline
\end{tabular}

Fonte: Autores (2021).

Abensur (2004), cita no seu trabalho que em todo paciente renal crônico que apresentar uma depuração de creatinina estimada menor que $60 \mathrm{~mL} / \mathrm{min} / 1,73 \mathrm{~m}^{2}$, é conveniente investigar a presença de anemia, o estoque de ferro, saturação de tranferrina, ferritina, vitamina B12 e folato, para que se possa identificar o tipo de anemia e estabelecer um tratamento adequado. Cita ainda que, $52 \%$ dos pacientes que se submetem à hemodiálise apresentam deficiência de ferro. De Miranda et al. (2018) estudaram 76 pacientes em tratamento dialítico com média de idade de 50,37 anos, e os resultados mostraram 80,3\% de anêmicos e $92,1 \%$ apresentaram VCM dentro da normalidade, isto é, tinham anemia normocítica. Barreto, Santos \& Vivas (2016) observaram $96 \%$ da amostra com valores de VCM dentro da normalidade.

\section{Conclusão}

A insuficiência renal é doença crônica limitante e traz como agravante comorbidades como anemia da doença renal crônica. Observa-se principalmente que é uma patologia de significância relevante e que acomete principalmente idosos do sexo masculino. A anemia é frequente e normalmente do tipo normocítica normocrômica. A creatinina é um biomarcador importante tanto para pacientes renais crônicos como para indivíduos saudáveis e pode ser utilizado como ferramenta coadjuvante no diagnóstico de disfunções renais. Inúmeros outros perfis podem ser pesquisados dos pacientes com insuficiência renal crônica, como a taxa de filtração glomerular relacionada com as formas possíveis de anemia e doenças renais associadas a anemias pré-existentes. Assunto amplo que permite várias abordagens na pesquisa e que traz colaboração importante para a ciência e pacientes acometidos por doenças renais.

\section{Referências}

Abensur, H. (2004). Anemia da Doença Renal Crônica. Brazilian Journal of Nephrology, 26 (3) 26-28.

Abensur, H. (2010). Deficiência de ferro na doença renal crônica. Revista Brasileira de Hematologia e Hemoterapia, 32 (2), 95 -98.

Abreu, A. P., Riella, M. C. \& Nascimento, M. M. (2020). The Brazilian Society of Nephrology and the Covid-19 Pandemic. Brazilian Journal of Nephrology, $42(2), 1-3$. 
Barreto, C. S., Santos, A. R. \& Vivas, W. L. P. (2016). Perfil hematológico em pacientes renais crônicos. Caderno de Graduação - Ciências Biológicas e da Saúde - UNIT SERGIPE, 3 (3), 149

Bastos, M. G., Carmo, W. D., Abrita, R. R., Almeida, E. D., Mafra, D., Costa, D. D., ... \& Paula, R. B. D. (2004). Doença renal crônica: problemas e soluções. Brazilian Journal of Nephrology, 26 (4), 202-215.

Bruce, A. \& Bruce, P. (2019). Estatística prática para cientistas de dados: 50 conceitos essenciais. Rio de Janeiro: Alta Books.

Bueno, C. S. \& Frizzo, M. N. (2014). Anemia na doença renal crônica em hospital da região noroeste do estado do Rio Grande do Sul. Jornal Brasileiro de Nefrologia, 36 (3), 304-314.

Bukabau, J. B., Yayo, E., Gnionsahé, A., Monnet, D., Pottel, H., Cavalier, E., ... \& Delanaye, P. (2019). Performance of creatinine- or cystatin C--based equations to estimate glomerular filtration rate in sub-Saharan African populations. Clinical Investigation, 95 (5), $1181-1189$.

Canziani, M. E. F., Bastos, M. G., Bregman, R., Pecoits Filho, R., Tomiyama, C., Draibe, S. A., \& Abensur, H. (2006). Deficiência de Ferro e Anemia na Doença Renal Crônica. Brazilian Journal of Nephrology, 28 (2), 86-90.

Crews, D. C., Bello, A. K. \& Saadi, G. (2019). Editorial do Dia Mundial do Rim 2019 - impacto, acesso e disparidades na doença renal. Brazilian Journal of Nephrology, 41 (1), 01-09.

De Miranda, D. E., Miranda, M. A. S. Q., Junior, P. R. S. E. \& Almeida, A. M. R. (2018) Prevalência de anemia nos pacientes com doença renal crônica em tratamento de hemodiálise. Brazilian Journal of Health Review, 1 (2), 282-296.

De Oliveira Filho, J.E.S. \& Caixeta, B.T. (2018). Incidência de anemia em pacientes com doença renal atendidos no laboratório do Hospital São Lucas no período de fevereiro a julho de 2014. Psicologia e Saúde em Debate, 4 (2) 1-13.

De Oliveira Junior, W. V., Zica, C. L. A., de Gouveia, I. P. P., de Oliveira Vasconcelos, M., Ramos, R. \& Oliveira, T. S. (2019). Anemia de doença crônica na doença renal crônica. Conexão Ci. Formiga MG, 14 (2), 57-65.

Draczevski, L. \& Teixeira, M. L. (2011). Avaliação do perfil bioquímico e hematológico em pacientes submetidos em hemodiálise. Revista Saúde e Pesquisa, $4(1), 15-22$.

Gordino, S. M. (2019). Epidemiologia da insuficiência renal crónica e anemia associada em adultos. Dissertação de mestrado, Universidade de Lisboa, Lisboa, Portugal. http://hdl.handle.net/10451/40606

Hoffbrand, A. V. \& Moss, P. A. H. (2018). Fundamentos em hematologia. Porto Alegre: Artmed.

Lehninger, T. M., Nelson, D.L. \& Cox, M. M. (2017). Princípios de bioquímica. São Paulo: Artmed

Negretti, C. D., Mesquita, P. G. M. \& Baracho, N. C. V. (2014). Perfil Epidemiológico de Pacientes Renais Crônicos em Tratamento Conservador em um Hospital Escola do Sul de Minas. Revista Ciências em Saúde, 4 (4), 49-60.

Núcleo Interdisciplinar de Estudo e Pesquisa em Nefrologia - NIEPEN. (2009). Doença renal crônica em pacientes idosos. Brazilian Journal of Nephrology, $31,59-65$.

Oliveira, R. A. G. (2011). Hemograma Cómo Hacer e Interpretar. Bogota: Actualidades Medico Odontologicas Latinoamerica.

Peixoto, A. B. S., Jaime, K. P.S. S., Melo Neto, J. R., Costa, S. H. N., Penna, K. G. B. D. \& Gomes, C. M. Avaliação de variáveis analíticas na doença renal crônica em pacientes atendidos em laboratórios de Goiânia - Goiás. Revista Brasileira Militar de Ciências, 5 (12), 15-21. doi: 10.36414/rbmc.v5i12.9.

Ribeiro, J. C. (2017). Prevalência de anemia em pacientes em tratamento hemodialítico e sua relação ao uso de eritropoetina e perfil clínicodemográfico. Universidade Federal de Uberlândia. https://repositorio.ufu.br/handle/123456789/21752.

Ribeiro-Alves, M. A. \& Gordan, P. A. (2014). Diagnóstico de anemia em pacientes portadores de doença renal crônica. Jornal Brasileiro de Nefrologia, 36 (1), 9-12.

Sarmento, L. R., Fernandes, P. F. C. B. C., Pontes, M. X., Correia, D. B. S., Chaves, V. C. B., Carvalho, C. F. D. A., ... \& Moliterno, L. A. A. (2018). Prevalence of clinically validated primary causes of end-stage renal disease (ESRD) in a State Capital in Northeastern Brazil. Brazilian Journal of Nephrology, 40 (2), 130-135.

Sesso, R. C., Lopes, A. A., Thomé, F. S., Lugon, J. R., \& Martins, C. T. (2017). Brazilian Chronic Dialysis Survey 2016. Jornal Brasileiro de Nefrologia, 39 (3) 261-266.

Silva, M. \& Couto, N. M. R. (2016). Avaliação do RDW como indicador da deficiência de ferro em pacientes com insuficiência renal crônica submetidos à hemodiálise. Revista Brasileira de Análises Clínicas, 48 (3) 211-5.

Soares, T. F. (2015). Perfil de pacientes renais crônicos com anemia submetidos à hemodiálise. Universidade de Brasília. https://bdm.unb.br/bitstream/10483/10895/1/2015_ThaisaFreireSoares.pdf.

Souza, P. D. P. \& Gomes, S. R. L. (2017). Anemia causada por deficiência de eritropoetina em pacientes renais crônicos. Revista Saber Científico, Porto Velho. URL: http://hdl.handle.net/123456789/2964 